\title{
C-reactive protein response to influenza vaccination as a model of mild inflammatory stimulation in the Philippines
}

\author{
Thomas W. McDade ${ }^{1,2,{ }^{*}}$, Judith B. Borja ${ }^{3}$, Christopher W. Kuzawa ${ }^{1,2}$, Tita Lorna L. Perez ${ }^{3}$, \\ and Linda S. Adair ${ }^{4}$ \\ ${ }^{1}$ Department of Anthropology: The Center on Social Disparities and Health Institute for Policy \\ Research Northwestern University Evanston, IL 60208 \\ ${ }^{2}$ Cells to Society (C2S): The Center on Social Disparities and Health Institute for Policy Research \\ Northwestern University Evanston, IL 60208 \\ ${ }^{3}$ Office of Population Studies Foundation University of San Carlos Cebu City, Philippines \\ ${ }^{4}$ Carolina Population Center and Department of Nutrition University of North Carolina, Chapel Hill \\ Chapel Hill, NC
}

\begin{abstract}
Background-C-reactive protein (CRP) is increasingly measured as a marker of systemic inflammation that predicts elevated risk for cardiovascular disease. Influenza vaccination is a mild pro-inflammatory stimulus, and the CRP response to vaccination may provide additional information on individual differences in inflammatory response and risk for disease.

Aim-To document the pattern of CRP response to influenza vaccination among a large sample of older women in the Philippines. The Philippines exemplifies current global trends toward increasing rates of overweight/obesity, but also maintains relatively high rates of infectious disease. The secondary aim of the study is to investigate the impact of infectious symptoms on the pattern of response to vaccination.
\end{abstract}

\begin{abstract}
Methods-A community-based sample of 934 women (mean age $=55.4$ years) received the influenza vaccine. CRP was assessed at baseline and 72 hours post-vaccination. Descriptive, nonparametric, and parametric analyses were implemented to assess the magnitude of CRP response, and to investigate whether responses were associated with baseline CRP or the presence of infectious symptoms prior to vaccination.
\end{abstract}

Results-Influenza vaccination resulted in a statistically significant CRP response of $0.35 \mathrm{mg} / \mathrm{L}$ ( $<<0.001$ ), representing a $30.2 \%$ increase from baseline. For individuals with symptoms of

\footnotetext{
(C) 2015 Published by Elsevier Ltd.

"Corresponding author Northwestern University Department of Anthropology 1810 Hinman Avenue Evanston, IL 60208 Phone: 847/467-4304 Fax: 847/467-1778 t-mcdade@ northwestern.edu.

Publisher's Disclaimer: This is a PDF file of an unedited manuscript that has been accepted for publication. As a service to our customers we are providing this early version of the manuscript. The manuscript will undergo copyediting, typesetting, and review of the resulting proof before it is published in its final citable form. Please note that during the production process errors may be discovered which could affect the content, and all legal disclaimers that apply to the journal pertain.

Conflict of Interest Statement: All authors declare that there are no conflicts of interest.
} 
infectious disease at baseline, the CRP response was smaller (12.9\%) and not statistically significant $(\mathrm{p}=0.77)$. Lower $\mathrm{CRP}$ at baseline was associated with larger CRP response to vaccination in the entire sample, and among participants without recent symptoms of infection.

Conclusions-Influenza vaccination produces a mild CRP response in the Philippines. This study extends prior research in US and European populations validating influenza vaccination as an in vivo model for investigating the dynamics of inflammation, but also raises potential complications in settings where rates of infectious disease are elevated.

\section{Keywords}

C-reactive protein; inflammation; influenza vaccination

\section{Introduction}

Inflammation has been implicated in multiple aspects of atherosclerosis, and C-reactive protein (CRP) is widely measured epidemiologically and clinically as an end-point marker of systemic inflammation that predicts elevated risk for incident cardiovascular disease [1], type 2 diabetes [2], late-life disability [3], and all-cause mortality [4-7]. As a prototypical acute phase protein, CRP is produced in response to pro-inflammatory cytokine signals, and concentrations increase acutely as part of innate immune responses to infection or injury [8-10]. Vaccination provides an opportunity to investigate individual differences in acute inflammatory responses which may provide additional information on disease risk beyond what can be obtained from baseline measures of systemic inflammation [11-13].

Recently, the influenza vaccine has been proposed as an in vivo model for probing mild stimulation of inflammatory processes. Among healthy older adults in the Netherlands [14] and the US [15], influenza vaccination resulted in small, but significant increases in CRP one to three days post-vaccination. In men with carotid artery disease, the CRP response to vaccination was 1.3 times greater than the response in healthy controls [16], and distinct patterns of response have been reported in patients with stable versus unstable forms of coronary heart disease [17]. Exaggerated inflammatory responses to influenza vaccination have also been associated with greater depressive symptoms in older adults [13], and among pregnant women [18]. These studies underscore the potential value of influenza vaccination as a mild, controlled stimulus for investigating individual differences in inflammatory responses in vivo.

The objective of this study is to document the pattern of CRP response to influenza vaccination among older women in the Philippines. Prior studies on the inflammatory response to vaccination, and on the associations among inflammation and cardiovascular disease more broadly, have been conducted primarily in high income American and European settings. Comparative data from a broader range of populations are needed since 85 percent of the global burden of cardiovascular disease now rests on the shoulders of low and middle income nations [19]. In addition, many of these nations are experiencing double burdens of disease: rising rates of cardiovascular and metabolic diseases are supplementing, rather than supplanting, infectious diseases as ongoing contributors to morbidity and mortality [20,21]. Given the important role CRP plays in the acute phase response to 
infection, a higher prevalence of infectious disease may complicate efforts to apply vaccination as an in vivo model of inflammatory response.

Lastly, the dynamics of inflammation may differ across populations in ways that influence the response to vaccination. For example, in prior work we reported that baseline CRP concentrations among healthy adults in the Philippines are significantly lower than in the US $[22,23]$, and that higher levels of microbial exposure in infancy may contribute to lower levels of chronic inflammation [24]. It is therefore important to investigate whether the CRP response to an inflammatory challenge is similar to what has been reported in similar studies in US and European contexts. Furthermore, prior research has indicated that the CRP response to vaccination is independent of baseline CRP [16], but whether this is a universal phenomenon is not known.

The Philippines is a lower-middle income nation that exemplifies current global trends toward increasing rates of overweight/obesity, cardiovascular diseases, and the metabolic syndrome as a result of economic, dietary, and lifestyle transitions [25-27]. At the same time, infectious disease remains a significant source of morbidity and mortality, with respiratory infections ranking along with ischemic heart disease as the top causes of death [28]. The specific objectives of this paper are threefold: 1) To document the magnitude of CRP response to influenza vaccination in a large sample of older adult women in the Philippines; 2) To investigate the impact of infectious symptoms prior to vaccination on the pattern of response; and 3) To evaluate the association between baseline CRP concentrations and the magnitude of vaccine response. Results from this study may shed light on the utility of influenza vaccination as an in vivo model for investigating the dynamics of inflammation among adults around the world.

\section{Materials and Methods}

\section{Participants and study design}

The Cebu Longitudinal Health and Nutrition Survey (CLHNS) began in 1983 with the recruitment of a community-based sample of 3327 pregnant women. The CLHNS objectives and survey design have been described previously [29]. The data for the present analyses come from the most recent survey, conducted between 2012-2013, which enrolled 1818 women, or $55 \%$ of the original sample. Of these, 967 were administered the influenza vaccine through intramuscular injection, using the 2012 Southern Hemisphere composition (Sanofi Pasteur, Vaxigrip single dose, $0.5 \mathrm{~mL}$ ). The vaccine study was implemented between April and December 2012, the period of time between the release of the vaccine in the Philippines and its expiration. Participants were screened by study physicians in local health centers prior to vaccination. Individuals with fever or other sickness on the day of vaccination were rescheduled. $\mathrm{N}=851(46.8 \%)$ were not vaccinated for the following reasons: Enrollment after vaccine expiration date $(n=316)$, refusal $(n=215)$, difficulty scheduling vaccination $(n=164)$, or ineligibility $(n=115$; recently vaccinated, allergic to egg/ chicken, or other health condition).

The final sample included $\mathrm{N}=934$ women with CRP results at baseline and day 3 . The average time between vaccination and day 3 blood sampling was 72.1 hours, with 95 percent 
of blood samples collected between 72.0 and 72.5 hours, and all samples collected between 71 and 74 hours post-vaccination. Baseline blood samples were collected immediately prior to vaccination (mean time between blood sampling and vaccination $=11.8$ minutes). All data were collected under conditions of informed consent with institutional review board approval from the University of North Carolina, Chapel Hill and the Office of Population Studies Foundation at the University of San Carlos, Cebu.

Blood samples were collected as finger stick capillary whole blood, dried on filter paper (dried blood spots, DBS) for the analysis of CRP. Each participant's finger was cleaned with alcohol, and a sterile, disposable microlancet was used to deliver a controlled, uniform puncture. Whole blood was placed directly on standardized filter paper commonly used for neonatal screening (Whatman \#903, GE Healthcare, Pascataway, NJ). This relatively noninvasive blood collection protocol minimizes pain and inconvenience to the participants, and facilitates the collection of repeat blood samples despite the constraints of field-based research settings [30]. After collection, DBS cards were allowed to dry at ambient temperatures for approximately four hours, and were stored in gas impermeable bags at $-20^{\circ} \mathrm{C}$ prior to shipment. Samples were express shipped to the U.S. where they were stored at $-30^{\circ} \mathrm{C}$ prior to analysis.

\section{Measurement of CRP}

Samples were analyzed for CRP in the Laboratory for Human Biology Research at Northwestern University using a modified high sensitivity enzyme immunoassay protocol previously developed for use with DBS [31]. Prior validation of assay performance indicates that the DBS CRP method produces results that are comparable to gold standard plasmabased clinical methods, with a lower limit of detection of $0.03 \mathrm{mg} / \mathrm{L}$ [31]. To minimize between-assay variation, all samples were analyzed by the same technician using a single lot of capture antibody, detection antibody, and calibration material. In addition, baseline and day 3 samples for each individual were included on the same assay plate in order to enhance within-individual comparisons. Between-assay CVs for low, mid, and high control samples included with all runs were $9.6 \%, 8.7 \%$, and $10.6 \%$, respectively.

Analysis of DBS samples provides concentrations of whole blood CRP, which will differ from plasma CRP due to the presence of lysed erythrocytes and associated matrix effects. However, since DBS and plasma results are highly correlated a conversion formula can be applied to DBS CRP results to calculate plasma equivalent values [32]. We converted DBS results into plasma equivalent values using a study-specific conversion formula based on $\mathrm{n}=69$ matched DBS and plasma samples, collected for a prior assay validation study. DBS samples were analyzed using the same procedures, lot number of reagents, and technician as applied to the study DBS samples. Plasma samples were analyzed for high sensitivity CRP in a high throughput clinical laboratory (NorthShore Research Institute), on the Beckman Coulter Synchron DXC platform. The correlation between DBS and serum values was high (Pearson $\mathrm{R}=0.98$ ) and the resulting Deming regression conversion formula was as follows: plasma $(\mathrm{mg} / \mathrm{L})=1.64 \times \mathrm{DBS}(\mathrm{mg} / \mathrm{L})$. 


\section{Data analysis}

The distribution of CRP values was highly skewed at baseline and day 3, and values were therefore log transformed (base 10) to normalize the distribution. However, descriptive analyses and statistical tests were implemented using untransformed as well as transformed results to confirm similar patterns of results. Non-parametric tests of differences in medians were implemented using Wilcoxon matched-pairs signed-ranks and rank sum tests, and Spearman rank-order correlation was used to assess strength of association. Log-transformed results were subjected to paired $t$ tests, Pearson correlation, and ordinary least squares regression analyses.

At baseline, prior to vaccination, participants were asked whether had been sick in the last week. Participants reported their symptoms, and a dichotomous variable indicating the presence of infectious symptoms was created with a value of 1 for any of the following symptoms: diarrhea, urinary tract infection, body aches, fever, cold, vomiting, pneumonia, sinusitis, tonsillitis, sore throat, or open wound. Individuals were not vaccinated if they had fever $\left(>37.5^{\circ} \mathrm{C}\right)$ or sickness on the day of vaccination; therefore this variable represents recent symptoms of infectious disease, in the week prior to vaccination. Sign rank and $t$ tests were implemented to investigate differences in CRP response to vaccination associated with the presence or absence of infectious symptoms at baseline.

Additional anthropometric data were collected using standard techniques [33], and demographic and health-related information was collected during baseline interviews to characterize the sample. A daily smoker was defined as one or more cigarettes/day, each day of the week. Participants were also asked to report any medications they were currently taking, and a summary variable indicating the use of anti-inflammatory medication was constructed based on the following MIMS sub-classes [34]: GIT regulators, antiflatulents, and anti-inflammatories; analgesics (non-opioid) and antipyretics; nonsteroidal antiinflammatory drugs (NSAIDs); corticosteroid hormones. Hormone replacement therapy $(\mathrm{N}=2)$ and statins $(\mathrm{N}=12)$ were not reported with sufficient frequency for analysis.

\section{Results}

\section{Magnitude of CRP response to vaccination}

The average age of participants was 55.4 years, with low rates of smoking (8.6\%) and antiinflammatory medication use (4.5\%) (Table 1). At the time of vaccination $7.0 \%$ of participants reported one or more symptoms of infectious disease.

For the entire sample, median CRP concentration at baseline was $1.16 \mathrm{mg} / \mathrm{L}$, which is in the low end of the range for "average risk" [4]. Median CRP three days post-vaccination was $1.51 \mathrm{mg} / \mathrm{L}$, which is an increase of $0.35 \mathrm{mg} / \mathrm{L}$, or $30.2 \%$ (Figure 1). Post-vaccination concentrations of CRP were significantly higher according to the Wilcoxon test for differences in medians $(\mathrm{z}=8.96, \mathrm{p}<0.001)$, and the paired $t$ test for differences in mean $\log \mathrm{CRP}(\mathrm{t}=10.26, \mathrm{p}<0.001)$. 


\section{Symptoms of infection and CRP response to vaccination}

Sixty five participants (7.0\%) reported symptoms of infectious disease during the week preceding vaccination, and as expected, infectious symptoms were associated with elevated CRP. Median CRP was $1.70 \mathrm{mg} / \mathrm{L}$ at baseline for participants reporting infectious symptoms, compared with $1.12 \mathrm{mg} / \mathrm{L}$ for participants without infectious symptoms ( $\mathrm{z}=$ $-3.312, \mathrm{p}<0.001)$. Participants with recent infectious symptoms did not differ from the rest of the sample with respect to age, level of education, waist circumference, smoking status, or urban residence (all $\mathrm{p}>0.28$ ). They were, however, almost three times more likely to have taken anti-inflammatory medication $(12.3 \%$ vs. $3.9 \%$, Pearson chi square $=9.92, \mathrm{p}<0.01)$ in the preceding week. However, this group was small: Only eight participants reported recent infectious symptoms and anti-inflammatory medication use.

In the absence of recent symptoms of infection, day 3 median CRP was $1.47 \mathrm{mg} / \mathrm{L}$, a $31.2 \%$ increase from $1.12 \mathrm{mg} / \mathrm{L}$ at baseline. Wilcoxon test for differences in medians $(\mathrm{z}=9.31$, $\mathrm{p}<0.001)$, and the paired $t$ test for differences in mean $\log \mathrm{CRP}(\mathrm{t}=10.46, \mathrm{p}<0.001)$, indicated that this was a statistically significant increase. Results were virtually identical when participants reporting anti-inflammatory medication use $(\mathrm{N}=34)$ were removed from the sample.

The CRP response to vaccination was attenuated among participants reporting recent infectious symptoms: median CRP at day 3 was $1.92 \mathrm{mg} / \mathrm{L}$, representing a $12.9 \%$ increase from the baseline median of $1.70 \mathrm{mg} / \mathrm{L}$. Tests for difference in median CRP $(\mathrm{z}=0.288$, $\mathrm{p}=0.77)$ and mean $\log \mathrm{CRP}(\mathrm{t}=0.84, \mathrm{p}=0.40)$ indicated that the increase in CRP following vaccination was not statistically significant for individuals reporting infectious symptoms. The pattern of CRP response was virtually identical when participants reporting antiinflammatory medication use $(\mathrm{N}=8)$ were removed.

\section{Baseline CRP and response to vaccination}

We next investigated whether baseline CRP concentration affected the magnitude of the CRP response to vaccination. Since recent symptoms of infectious disease were associated with elevated CRP at baseline, we removed participants with infectious symptoms from these analyses. We divided the sample into baseline CRP groups according to the distribution of values in the sample. We then determined the CRP response for each individual $\left(\mathrm{CRP}_{\text {Day } 3}-\mathrm{CRP}_{\text {baseline }}\right)$ and calculated the median response within each group in $\mathrm{mg} / \mathrm{L}$, and as a percentage of baseline $\left(\mathrm{CRP}_{\text {response }} / \mathrm{CRP}_{\text {baseline }}\right)$ (Table 2$)$. When baseline CRP was $\leq 3.0$, the response to vaccination was positive and ranged from 0.10 to $0.32 \mathrm{mg} / \mathrm{L}$. At higher concentrations of baseline CRP, the median response was negative, with substantially lower CRP at Day 3.

At baseline, $22.4 \%$ of the sample had CRP $\leq 0.5 \mathrm{mg} / \mathrm{L}$, and the median response to vaccination was relatively low at $0.10 \mathrm{mg} / \mathrm{L}$. However, when considered as a percentage of baseline, the magnitude of response was $34.0 \%$. The percent response was $38.9 \%$ for baseline CRP > 0.5 to $\leq 1.0$, and then decreased with higher baseline CRP and became negative for CRP > $3.0 \mathrm{mg} / \mathrm{L}$. Spearman's rank correlation confirms that higher baseline 
CRP is significantly associated with lower CRP response to vaccination, in terms of $\mathrm{mg} / \mathrm{L}$ $($ rho $=-0.12, \mathrm{p}<0.001)$ and percent response $($ rho $=-0.34, \mathrm{p}<0.001)$.

We confirmed this pattern of results with $\log C R P_{\text {response, }}$, which is negatively correlated with $\log C R P_{\text {baseline }}($ Pearson $\mathrm{R}=-0.33, \mathrm{p}<0.001$ ). In regression models we added a quadratic term $\left(\log C \mathrm{RP}_{\text {baseline }}{ }^{2}\right)$ since the results above suggested the possibility of a nonlinear association between baseline CRP and vaccine response. $\operatorname{LogCRP} P_{\text {baseline }}$ is a significant predictor of $\log C R P_{\text {response }}(B=-0.205, S E=0.020, p<0.001)$, as is the quadratic term $(\mathrm{B}=-0.054, \mathrm{SE}=0.027, \mathrm{p}<0.05)$. As shown in figure $2, \mathrm{CRP}$ response to vaccination is highest at low baseline CRP concentrations, and becomes increasingly attenuated with higher baseline CRP. The pattern of association between baseline CRP and response to vaccination was virtually identical when individuals with recent symptoms of infectious disease were included. Similarly, when we added the variables in Table 1 as predictors of $\log C R P_{\text {response, }}$, the pattern of association with $\log C R P_{\text {baseline }}$ was unchanged. In addition to $\log \mathrm{CRP}_{\text {baseline, }}$, waist circumference was a significant predictor of vaccine response $(\mathrm{B}=$ $0.002, \mathrm{SE}=0.001, \mathrm{p}<0.05)$.

\section{Discussion}

Dynamic measures of inflammation may provide important insights into individual differences in the regulation of inflammation and risk for disease [11,14,16]. Results from this study extend prior research in affluent US and European populations validating influenza vaccination as an in vivo model for investigating the inflammatory response to mild stimulation. We confirm that influenza vaccination induces a mild acute inflammatory response, but we also report attenuated responses in relation to infectious symptoms and higher baseline CRP, findings that may have important implications for applying the vaccine model in future research, particularly in epidemiologic settings with elevated rates of infectious disease.

In our sample of older women in the Philippines the pattern of CRP response to vaccination is similar in magnitude to prior research [14-16]. The increase in CRP is modest but significant at $0.35 \mathrm{mg} / \mathrm{L}$, representing a $30.2 \%$ increase from baseline. This response is larger than the $0.20 \mathrm{mg} / \mathrm{L}$ increase reported in the Netherlands [14], and smaller than the $40-63 \%$ increase among older adults in two recent studies in the US $[15,16]$.

To the best of our knowledge, this study is the first to document differences in CRP response to influenza vaccination in relation to symptoms of infectious disease and baseline concentrations of CRP. In their study of 38-88 year-old men, Carty et al. [16] report no association between baseline CRP and the magnitude of response to vaccination. We find a significant negative association, with the highest CRP responses among participants with lower CRP at baseline. We also find attenuated, and statistically insignificant, responses among participants reporting symptoms of infectious disease in the week preceding vaccination.

It is well established that CRP concentrations increase as part of the acute phase response to a wide range of pathogens $[8,35]$, and the CRP response to vaccination may therefore be 
obscured in participants with prior infectious symptoms. A similar process may explain the negative association between baseline CRP and vaccine response: Although the reported frequency of infectious symptoms in our sample was $7.0 \%$, it is likely that the level of subclinical infection was substantially higher. If elevated CRP at baseline was due to the presence of infectious symptoms - reported or not-then the negative association between baseline CRP and response to vaccination may reflect resolution of an active acute phase response that was in process the week preceding vaccination.

Alternatively, the CRP response to vaccination may provide insight into stable individual differences in the regulation of inflammation. The combination of low baseline CRP and robust increases in response to challenge may represent the optimal pattern, whereby acute responsiveness to infection is maximized but risk for diseases associated with chronic inflammation is minimized. In contrast, lack of response to vaccination may signal a poorly regulated inflammatory system that increases risk for disease. Previously, we have shown that higher levels of microbial exposure during infancy predict lower levels of chronic inflammation in the Philippines [24,36], consistent with a wider literature documenting the importance of microbial exposure to the development of immunoregulatory networks during critical periods of development [37-39]. In this context, it is interesting to consider whether the absence of correlation between baseline CRP and vaccine response reported in the US reflects a distinct pattern of regulation that is the result of lower levels of microbial exposure in infancy and higher levels of chronic inflammation in adulthood.

Limitations of the study include the exclusive focus on women, and the use of a single postvaccination CRP measure. Prior research has suggested that the inflammatory response to vaccination may peak at 48 hours [14], although other studies have followed up after 24 or 72 hours [15,16], and it would have been preferable to have multiple measures of response over time. A major strength is the large, community-based sample which enhances external validity, and provides opportunities for investigating the long term association between CRP responsiveness and risk for disease. Follow up studies will be particularly important for determining if measures of inflammatory response provide predictive value above and beyond currently recommended measures of baseline chronic inflammation [4].

\section{Acknowledgments}

Funding statement: Research reported in this manuscript was supported by the National Institute of Aging of the National Institutes of Health under award number R01AG039443. This research also received support from the Population Research Infrastructure Program awarded to the Carolina Population Center (R24 HD050924) at The University of North Carolina at Chapel Hill by the Eunice Kennedy Shriver National Institute of Child Health and Human Development. The content is solely the responsibility of the authors and does not necessarily represent the official views of the National Institutes of Health.

\section{References}

1. Ridker PM, Buring JE, Shih J, Matias M, Hennekens CH. Prospective study of C-reactive protein and the risk of future cardiovascular events among apparently healthy women. Circulation. 1998; 98:731-3. [PubMed: 9727541]

2. Pradhan AD, Manson JE, Rifai N, Buring JE, Ridker PM. C-reactive protein, interleukin 6, and risk of developing type 2 diabetes mellitus. Journal of American Medical Association. 2001; 286:32734. 
3. Kuo H-K, Bean JF, Yen C-J, Leveille SG. Linking c-reactive protein to late-life disability in the National Health and Nutrition Examination Survey (NHANES) 1999-2002. The Journals of Gerontology Series A: Biological Sciences and Medical Sciences. 2006; 61:380-7.

4. Pearson TA, Mensah GA, Alexander RW, et al. Markers of inflammation and cardiovascular disease: Application to clinical and public health practice. Circulation. 2003; 107:499-511. [PubMed: 12551878]

5. Berg AH, Scherer PE. Adipose tissue, inflammation, and cardiovascular disease. Circ Res. 2005; 96:939-49. [PubMed: 15890981]

6. Jenny NS, Yanez ND, Psaty BM, Kuller LH, Hirsch CH, Tracy RP. Inflammation biomarkers and near-term death in older men. American Journal of Epidemiology. 2007; 165:684-95. [PubMed: 17215383]

7. Harris TB, Ferrucci L, Tracy RP, et al. Associations of elevated interleukin-6 and C-reactive protein levels with mortality in the elderly. American Journal of Medicine. 1999; 106:516-2.

8. Ballou SP, Kushner I. C-reactive protein and the acute phase response. Advances in Internal Medicine. 1992; 37:313-36. [PubMed: 1558000]

9. Black S, Kushner I, Samols D. C-reactive protein. Journal of Biological Chemistry. 2004; 279:48487-90. [PubMed: 15337754]

10. Du Clos TW. Function of C-reactive protein. Annals of Medicine. 2000; 32:274-8. [PubMed: 10852144]

11. Christian LM. Psychoneuroimmunology in pregnancy: Immune pathways linking stress with maternal health, adverse birth outcomes, and fetal development. Neurosci Biobehav Rev. 2012; 36:350-61. [PubMed: 21787802]

12. El Yousfi M, Mercier S, Breuille D, et al. The inflammatory response to vaccination is altered in the elderly. Mech Ageing Dev. 2005; 126:874-81. [PubMed: 15876450]

13. Glaser R, Robles TF, Sheridan J, Malarkey WB, Kiecolt-Glaser JK. Mild depressive symptoms are associated with amplified and prolonged inflammatory responses after influenza virus vaccination in older adults. Arch Gen Psychiatry. 2003; 60:1009-14. [PubMed: 14557146]

14. Posthouwer D, Voorbij HA, Grobbee DE, Numans ME, van der Bom JG. Influenza and pneumococcal vaccination as a model to assess C-reactive protein response to mild inflammation. Vaccine. 2004; 23:362-5. [PubMed: 15530681]

15. Tsai MY, Hanson NQ, Straka RJ, et al. Effect of influenza vaccine on markers of inflammation and lipid profile. J Lab Clin Med. 2005; 145:323-7. [PubMed: 15976761]

16. Carty CL, Heagerty P, Nakayama K, et al. Inflammatory response after influenza vaccination in men with and without carotid artery disease. Arterioscler Thromb Vasc Biol. 2006; 26:2738-44. [PubMed: 17023683]

17. Werba JP, Veglia F, Amato M, et al. Patients with a history of stable or unstable coronary heart disease have different acute phase responses to an inflammatory stimulus. Atherosclerosis. 2008; 196:835-40. [PubMed: 17335831]

18. Christian LM, Franco A, Iams JD, Sheridan J, Glaser R. Depressive symptoms predict exaggerated inflammatory responses to an in vivo immune challenge among pregnant women. Brain Behav Immun. 2010; 24:49-53. [PubMed: 19464358]

19. Yusuf S, Reddy S, Ounpuu S, Anand S. Global burden of cardiovascular diseases: Part i: General considerations, the epidemiologic transition, risk factors, and impact of urbanization. Circulation. 2001; 104:2746-53. [PubMed: 11723030]

20. Barrett RL, Kuzawa CW, McDade TW, Armelagos GJ. Emerging and re-emerging infectious diseases: The third epidemiological transition. Annual Review of Anthropology. 1998; 27:247-71.

21. Boutayeb A. The double burden of communicable and non-communicable diseases in developing countries. Transactions of the Royal Society of Tropical Medicine and Hygiene. 2006; 100:191-9. [PubMed: 16274715]

22. McDade T, Rutherford JN, Adair L, Kuzawa C. Adiposity and pathogen exposure predict Creactive protein in Filipino women. Journal of Nutrition. 2008; 138:2442-7. [PubMed: 19022970]

23. McDade T, Rutherford JN, Adair L, Kuzawa C. Population differences in c-reactive protein concentration and associations with adiposity: Comparing young adults in the Philippines and the U.S. American Journal of Clinical Nutrition. 2009; 89:1237-45. [PubMed: 19225115] 
24. McDade TW, Rutherford J, Adair L, Kuzawa CW. Early origins of inflammation: Microbial exposures in infancy predict lower levels of C-reactive protein in adulthood. Proc Biol Sci. 2010; 277:1129-37. [PubMed: 20007176]

25. Adair LS. Dramatic rise in overweight and obesity in adult Filipino women and risk of hypertension. Obesity Research. 2004; 12:1335-41. [PubMed: 15340117]

26. Tanchoco CC, Cruz AJ, Duante CA, Litonjua AD. Prevalence of metabolic syndrome among Filipino adults aged 20 years and over. Asia Pacific J Clin Nutr. 2003; 12:271-6.

27. Pedro MR, Barba CVC, Benavides-de Leon R. Nutrition transition in the Philippines. Philippine Population Review. 2007; 6:1-19.

28. WHO. Mortality country fact sheet 2006. World Health Organization; 2006.

29. Adair LS, Popkin BM, Akin JS, et al. Cohort profile: The Cebu Longitudinal Health and Nutrition Survey. Int J Epidemiol. 2011; 40:619-25. [PubMed: 20507864]

30. Mcdade TW, Williams S, Snodgrass JJ. What a drop can do: Dried blood spots as a minimally invasive method for integrating biomarkers into population-based research. Demography. 2007; 44:899-925. [PubMed: 18232218]

31. McDade TW, Burhop J, Dohnal J. High-sensitivity enzyme immunoassay for C-reactive protein in dried blood spots. Clin Chem. 2004; 50:652-4. [PubMed: 14981035]

32. McDade TW. Development and validation of assay protocols for use with dried blood spot samples. Am J Hum Biol. 2014; 26:1-9. [PubMed: 24130128]

33. Lohman, TG.; Roche, AF.; Martorell, R. Anthropometric standardization reference manual. Human Kinetics Books; Champaign, IL: 1988.

34. MIMS Philippines: Philippine index of medical specialties. 123rd edition.. CMPMedia Asia Pte Ltd.; Singapore: 2010.

35. Nakayama T, Sonoda S, Urano T, Yamada T, Okada M. Monitoring both serum amyloid protein A and C-reactive protein as inflammatory markers in infectious diseases. Clin Chem. 1993; 39:2937. [PubMed: 8381732]

36. McDade TW. Early environments and the ecology of inflammation. Proceedings of the National Academy of Sciences. 2012; 109:17281-8.

37. Yazdanbakhsh M, Dremsner PG, van Ree R. Allergy, parasites, and the hygiene hypothesis. Science. 2002; 296:490-4. [PubMed: 11964470]

38. Lisciandro JG, van den Biggelaar AH. Neonatal immune function and inflammatory illnesses in later life: Lessons to be learnt from the developing world? Clin Exp Allergy. 2010; 40:1719-31. [PubMed: 20964742]

39. Rook GAW. Review series on helminths, immune modulation and the hygiene hypothesis: The broader implications of the hygiene hypothesis. Immunology. 2009; 126:3-11. [PubMed: 19120493] 


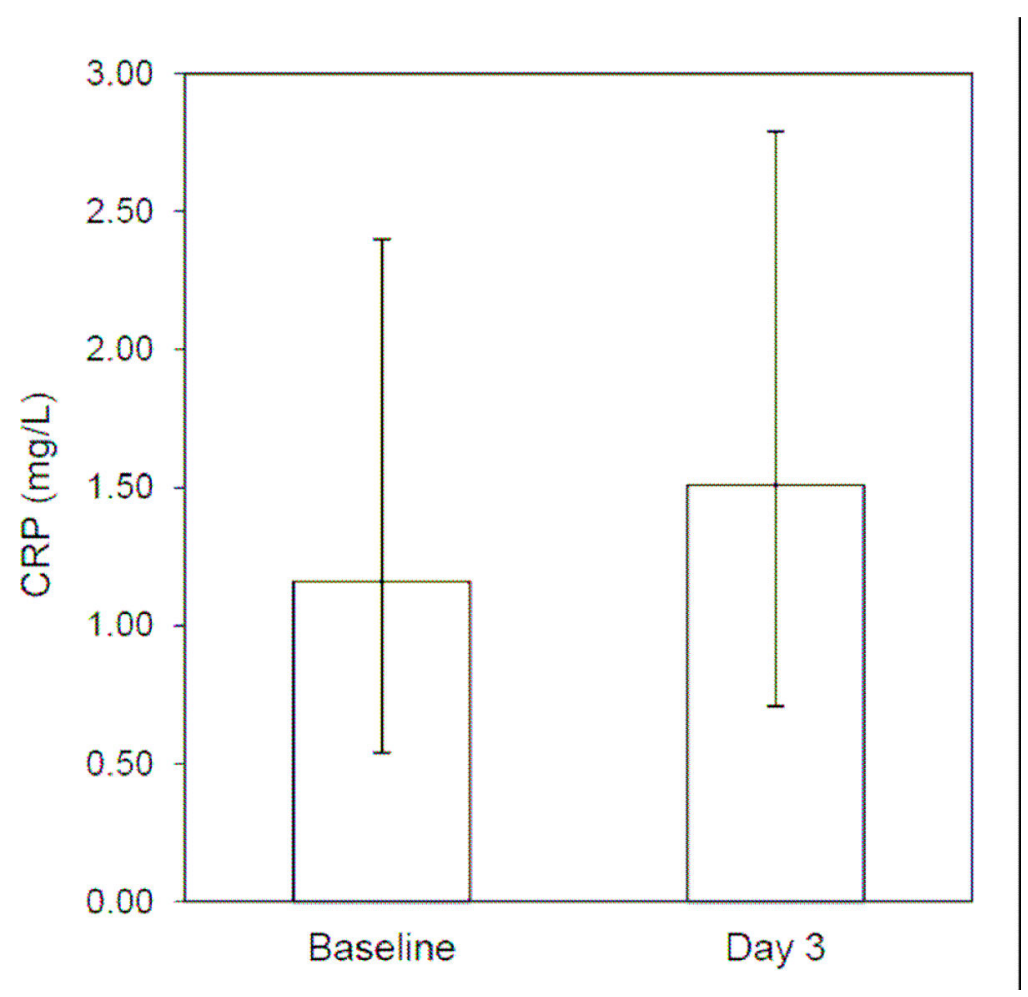

Figure 1.

CRP response to influenza vaccination. Median concentrations of CRP at baseline and three days following vaccination (error bars represent $25^{\text {th }}$ and $75^{\text {th }}$ percentile values). 


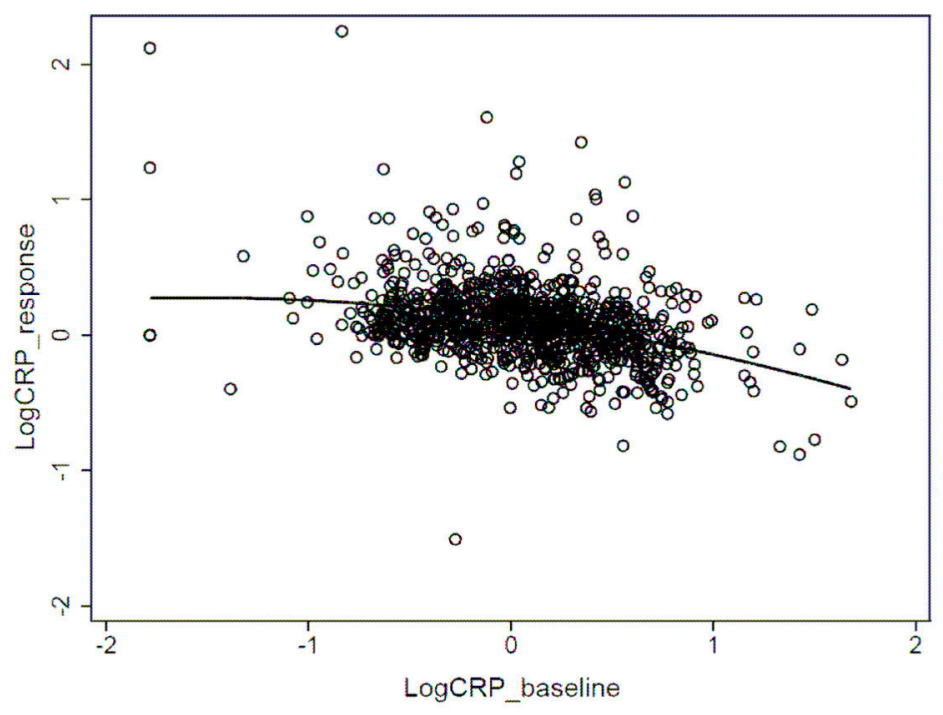

Note: Best fit line is based on the following regression equation $\left(p<0.001, R^{2}=0.12\right)$ :

$\operatorname{LogCRP} P_{\text {response }}=-0.205\left(\log C R P_{\text {baseline }}\right)-0.054\left(\operatorname{LogCRP}\right.$ baseline $\left.^{2}\right)+0.12$.

Figure 2.

Scatterplot of CRP response to vaccination in relation to baseline CRP concentration. 


\section{Table 1}

Baseline characteristics for study participants ( $\mathrm{n}=934)$.

\begin{tabular}{ll}
\hline & Total \\
Age (years) & $55.4 \pm 5.9$ \\
Education (highest grade) & $7.1 \pm 3.5$ \\
Waist circumference (cm) & $81.9 \pm 11.1$ \\
Urban residence (\%) & 82.6 \\
Daily smoker (\%) & 8.6 \\
Current infection (\%) & 7.0 \\
Anti-inflammatory medication (\%) & 4.5 \\
\hline
\end{tabular}

Values are mean \pm standard deviation for continuous variables. 
Table 2

CRP response to vaccination in relation to baseline CRP concentrations ( $\mathrm{N}=869$; participants with recent symptoms of infection excluded from analysis).

\begin{tabular}{llll}
\hline Baseline CRP & $\mathbf{N}$ & median CRP response, $\mathbf{m g} / \mathbf{L}$ (range) & median CRP response, \% of baseline (range) \\
$\unlhd .5 \mathrm{mg} / \mathrm{L}$ & 195 & $0.10(-0.19,25.55)$ & $34.0(-60.0,17504.5)$ \\
$>0.5$ to $\leq 1.0$ & 194 & $0.28(-0.70,30.01)$ & $38.9(-96.9,3960.6)$ \\
$>1.0$ to $\_.0$ & 223 & $0.32(-1.13,19.76)$ & $24.6(-70.8,1806.4)$ \\
$>2.0$ to $\leq 3.0$ & 109 & $0.24(-1.68,56.6)$ & $10.1(-71.1,2553.3)$ \\
$>3.0$ to $\leq .0$ & 93 & $-0.27(-3.01,45.45)$ & $-6.0(-68.8,1245.4)$ \\
$>5.0$ to $\leq 10.0$ & 44 & $-1.39(-4.83,7.55)$ & $-19.6(-73.8,110.9)$ \\
$>10.0$ & 11 & $-8.35(-32.41,13.62)$ & $-34.1(-85.0,88.1)$ \\
\hline
\end{tabular}

